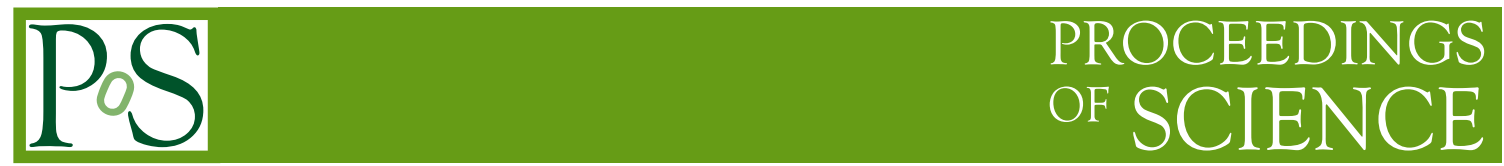

\title{
MultiJet Predictions for Higgs Studies
}

\section{J. R. ANDERSEN ${ }^{* a}$ and J. M. SMILLIE ${ }^{b}$}

a Institute for Particle Physics Phenomenology, Department of Physics, Durham University Durham, DH1 3LE, UK

${ }^{b}$ Higgs Centre for Theoretical Physics, School of Physic and Astronomy, University of Edinburgh Mayfield Road, Edinburgh, EH9 3JZ, UK

E-mail: jeppe.andersen@cern.ch, j.m.smillie@ed.ac.uk

We discuss several issues which arise when describing the production of multiple hard jets $(\geq 2)$ in LHC collisions and describe the solution offered through the High Energy Jets formalism. Throughout, there is particular emphasis on what we can and must learn through these descriptions in order to apply it to Higg-plus-dijet analyses.

XXI International Workshop on Deep-Inelastic Scattering and Related Subjects 22-26 April, 2013

Marseilles, France

\footnotetext{
*Speaker.
} 


\section{Multi-Jet Production}

Multi-jet processes are a key component of the LHC physics programme. Whilst also providing key tests of our understanding of QCD at the higher energies accessible at the LHC, the production of dijets and $W, Z$-plus-dijets take on particular significance in the light of the forthcoming Higgs-plus-dijets experimental analyses. Jet radiation patterns between these processes are observed to be similar, see figure 1. This shows the normalised distribution for the rapidity difference between the jets at leading-order for dijets, $W+$ dijets and Higgs+dijets. While there are

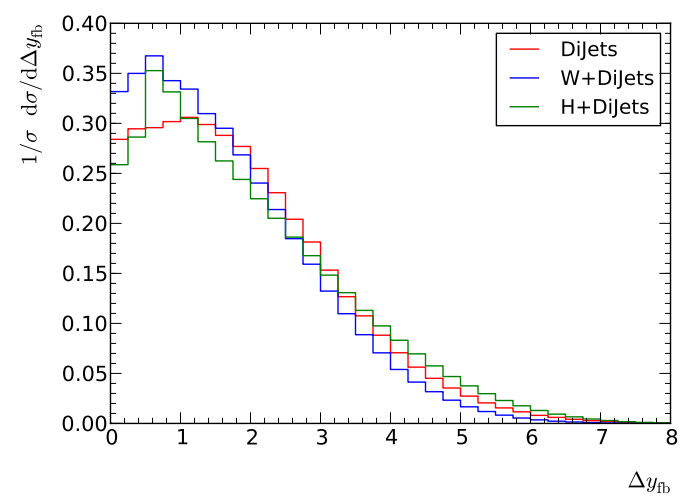

Figure 1: Normalised rapidity-difference distributions for dijets, $W+$ dijets and $H+$ dijets at leading order. In all three cases, all jets are required to satisfy $p_{T}>30 \mathrm{GeV}$ and $\left|\eta_{j}\right|<4.4$.

some differences at the lowest values of rapidity difference, the distributions are all extremely close above around $\Delta y=1.5$. This means that strategies designed for Higgs+dijets analyses can be studied and validated in the non-Higgs processes first, where there is already ample data. These strategies include the application of vector-boson-fusion (VBF) cuts and the use of a jet veto.

However, the theoretical description of multi-jet processes presents many challenges. For example, they contain many different scales (such as jet transverse momenta, invariant masses, vector boson masses). Furthermore, higher-order perturbative corrections to these processes can be large, especially in key regions of phase space. For example, it was observed in Ref. [1] that in order to describe data at large $H_{T}$, it was necessary to combine NLO samples at different orders. The inclusion of just one higher order in the perturbative series was not sufficient.

The High Energy Jets (HEJ) formalism [2,3] provides an alternative description of multi-jet processes which is built from an all-order description of (hard) wide-angle QCD radiation and therefore immediately includes the dominant contributions at all orders in $\alpha_{s}$.

\section{High Energy Jets}

HEJ is inspired by the behaviour of scattering amplitudes in the high energy limit. In this limit, the matrix elements simplify sufficiently to allow an efficient integration over large numbers of partons. This includes the amplitudes for virtual processes and, all together, gives a description which is accurate to leading-log in $s / t$. Naturally, there is some remaining freedom in how to describe the sub-leading contributions to the amplitudes. In HEJ, we use this freedom to describe 
all amplitudes as contractions of currents which means that the description is exact in the case of two final-state partons. For example, in the case of $q\left(p_{a}\right) Q\left(p_{b}\right) \longrightarrow q\left(p_{1}\right) g\left(p_{2}\right) \ldots g\left(p_{n-1}\right) Q\left(p_{n}\right)$,

$$
\begin{aligned}
\left.\overline{\mathscr{M}}_{q Q \rightarrow q g \cdot g Q}\right|^{2}= & \frac{1}{4\left(N_{C}^{2}-1\right)}\left\|S_{q Q \rightarrow q Q}\right\|^{2} \cdot\left(g^{2} C_{F} \frac{1}{t_{1}}\right) \cdot\left(g^{2} C_{F} \frac{1}{t_{n-1}}\right) \\
& \cdot \prod_{i=1}^{n-2}\left(\frac{-g^{2} C_{A}}{t_{i} t_{i+1}} V^{\mu}\left(q_{i}, q_{i+1}\right) V_{\mu}\left(q_{i}, q_{i+1}\right)\right) \cdot \prod_{j=1}^{n-1} \exp \left[\omega^{0}\left(q_{j}\right)\left(y_{j-1}-y_{j}\right)\right],
\end{aligned}
$$

where $q_{1}=p_{a}-p_{1}, q_{i+1}=q_{i}-p_{i}, t_{i}=q_{i}^{2}$,

$$
\left\|S_{q Q \rightarrow q Q}\right\|^{2}=\sum_{\text {helicities }}\left|j^{\mu}\left(p_{1}, p_{a}\right) j_{\mu}\left(p_{n}, p_{b}\right)\right|^{2}
$$

$V^{\mu}\left(q_{i}, q_{i+1}\right)$ is the effective vertex for the emission of a gluon of momentum $p_{2}$ and the exponentials encode the finite remainder after the cancellation of real and virtual divergences. Full details of these terms may be found in Ref. [2]. For channels with incoming gluons, one can still describe the process in terms of currents while is again exact for $q g \rightarrow q g$ [3].

The extension of this to include the production of a $W, Z / \gamma^{*}$ or Higgs boson in association with jets is achieved by modifying the currents in eq. (2.2). As an example, in order to describe the process $u g \rightarrow u g \ldots g\left(W^{+} \rightarrow\right) e^{+} v_{e}$, the only necessary modifcation is $j^{\mu}\left(p_{1}, p_{a}\right) \longrightarrow$ $j_{W}^{\mu}\left(p_{1}, p_{e}, p_{v}, p_{a}\right)$, where $j_{W}^{\mu}$ sums the contributions from emitting the $W$ at different points on the quark line. HEJ describes the production of Higgs-plus-dijets in the gluon-gluon fusion channel where the events have a vector boson fusion topology. In this channel in the heavy top-mass limit, the Higgs boson couples to two gluons of momentum $p^{\mu}$ and $q^{v}$ through an effective vertex of the form

$$
V_{H}^{\mu v}(p, q)=g^{\mu v} p \cdot q-p^{v} q^{\mu} .
$$

Within HEJ, then, if the Higgs boson is inserted at the correct point in the rapidity-ordered chain, with $q_{H 1}$ and $q_{H 2}$ on either side, $\left\|S_{q Q \rightarrow q Q}\right\|^{2}$ becomes

$$
\left\|S_{q Q \rightarrow q Q}^{H}\right\|^{2}=\sum_{\text {helicities }}\left|j_{\mu}\left(p_{1}, p_{a}\right) V_{H}^{\mu v}\left(q_{H 1}, q_{H 2}\right) j_{v}\left(p_{n}, p_{b}\right)\right|^{2} .
$$

The rest of the description remains the same. HEJ is available as a fully-flexible publicly-available Monte Carlo for the production of multiple jets [4], a $W$ boson in association with jets [5] and, most recently, a Higgs boson in association with jets. These programs and further details may all be obtained from http: //cern. ch/hej.

Sample results for the production of a Higgs boson with at least two jets are shown in figure 2 . The left panel shows the distribution of the transverse momentum of the Higgs boson which is less sensitive to additional radiation. The right-hand plot shows the distribution of the angular variable defined in Ref. [6] to study the azimuthal correlation between the jets in multi-jet events. The angle is measured between the vector sum of jets forward and jets backward of the Higgs boson. The peaks in the distribution clearly show the $\mathrm{CP}$-structure of the $g g H$-coupling.

\section{Higgs Plus Dijets within Vector Boson Fusion Cuts}

To reach the full potential of Higgs-plus-dijets measurements will require thorough testing and validation of the available theoretical descriptions of this process. First steps in this direction have 

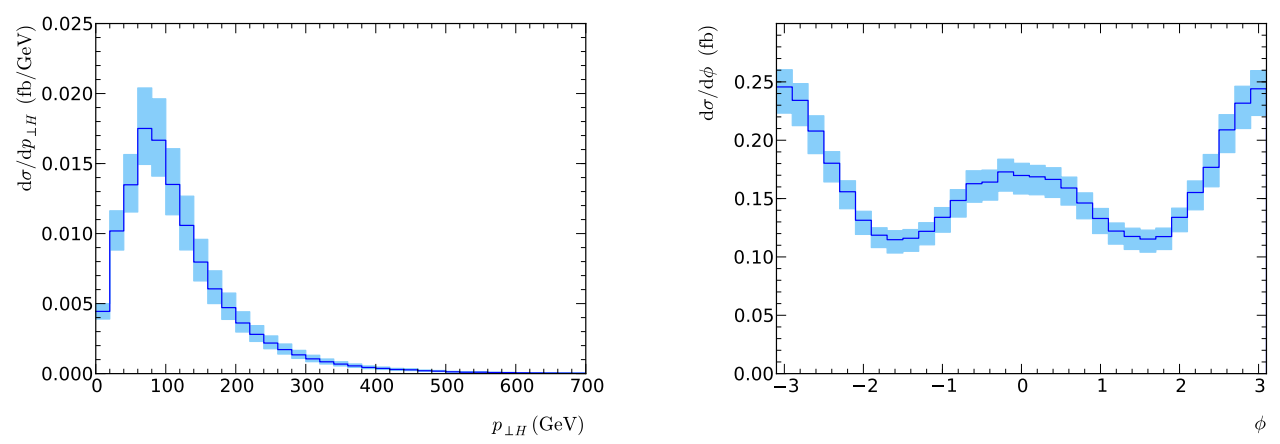

Figure 2: Differential distributions for the production of a Higgs boson with at least two jets. Jets are required to satisfy $p_{T}>30 \mathrm{GeV},|y|<4.5$ and also $\left|y_{H}\right|<2.5$. The left-hand side shows the distribution of $p_{T H}$. The right-hand side shows the distribution of the $\phi$-variable of Ref. [6]. The peaks at $0, \pm \pi$ arise from the CP-structure of $V_{H}$.

been taken in the recent third yellow report of the Higgs Cross Section Working Group [7]. Sample plots taken from this are shown in figure 3 showing the rapidity distribution of the leading jet. In both plots, the (anti-kt, $\mathrm{R}=0.4$ ) jets are required to satisfy $p_{T}>20 \mathrm{GeV}$ and $|y|<5$. In addition, on the right-hand plot, the two hardest jets are required to satisfy the following Vector Boson Fusion (VBF) cuts which are designed to select the distinct topology of these events:

$$
\left|\eta_{j 1}-\eta_{j 2}\right|>2.8, \quad m_{j 1, j 2}>400 \mathrm{GeV} .
$$

The impact of these cuts on the shape of the distribution is clear: the requirement of a rapidity separation leads to the double peaks seen in the right plot. The impact of these cuts has a greater effect on the total HEJ cross section (6\%) compared with the fixed-order based descriptions where the impact is roughly $10 \%$. Where more hard jets are present, the average rapidity difference between them will be less so the cut will have more impact. Jet radiation patterns were considered in the context of $W$-plus-jets in a recent D0 study [8]. Figure 4 shows that the leading-order based descriptions underestimated jet activity as the rapidity separation between the hardest two jets was increased. The NLO prediction combined $W+2$-jet and $W+3$-jet NLO samples.
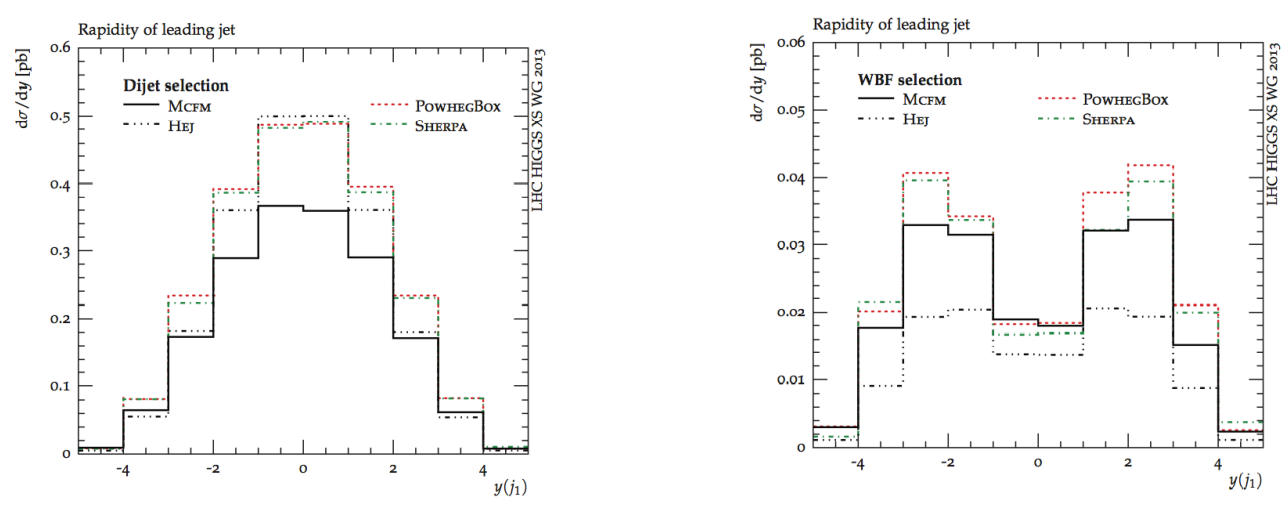

Figure 3: Sample plots taken from Ref. [7]. The rapidity of the leading jet is shown before (left) and after (right) imposing the VBF cuts of eq. (3.1). 


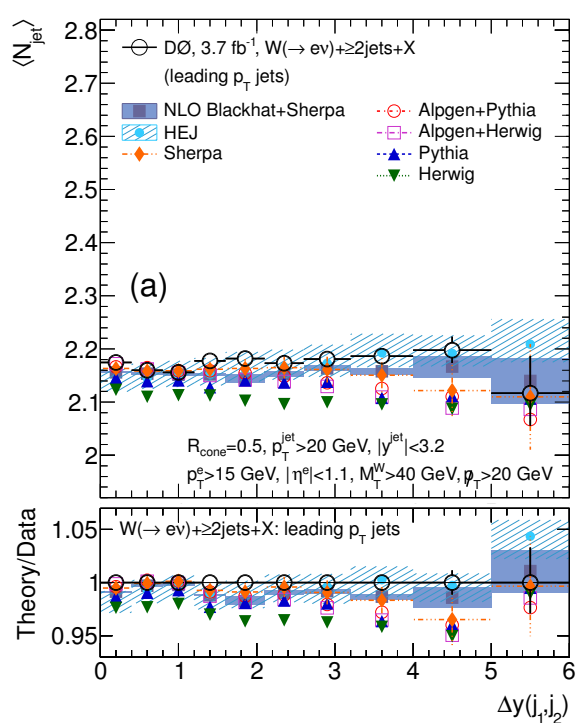

Figure 4: The average number of jets seen in inclusive $W+2$-jet events at D0, shown as a function of the rapidity difference between the two hardest jets. Plot taken from [8].

There has been a lot of significant progress in our ability and techniques to describe multi-jet processes. However, further work and studies are required to validate and understand the differences between the theoretical predictions in this key process to allow us to extract as much information as possible from the data.

\section{References}

[1] G. Aad et al. [ATLAS Collaboration], Study of jets produced in association with a W boson in $p p$ collisions at $\sqrt{s}=7$ TeV with the ATLAS detector, Phys. Rev. D 85 (2012) 092002, [arXiv:1201.1276 [hep-ex]].

[2] J. R. Andersen and J. M. Smillie, Constructing All-Order Corrections to Multi-Jet Rates, JHEP 1001 (2010) 039, [arXiv:0908.2786 [hep-ph]].

[3] J. R. Andersen and J. M. Smillie, The Factorisation of the t-channel Pole in Quark-Gluon Scattering, Phys. Rev. D 81 (2010) 114021, [arXiv:0910.5113 [hep-ph] ].

[4] J. R. Andersen and J. M. Smillie, Multiple Jets at the LHC with High Energy Jets, JHEP 1106 (2011) 010, [arXiv:1101.5394 [hep-ph]].

[5] J. R. Andersen, T. Hapola and J. M. Smillie, W Plus Multiple Jets at the LHC with High Energy Jets, JHEP 1209 (2012) 047, [arXiv:1206.6763 [hep-ph] ].

[6] J. R. Andersen, K. Arnold and D. Zeppenfeld, Azimuthal Angle Correlations for Higgs Boson plus Multi-Jet Events, JHEP 1006 (2010) 091 [arXiv:1001.3822 [hep-ph] ].

[7] S. Heinemeyer et al. [ The LHC Higgs Cross Section Working Group Collaboration], Handbook of LHC Higgs Cross Sections: 3. Higgs Properties, [arXiv:1307.1347 [hep-ph]].

[8] V. M. Abazov et al. [D0 Collaboration], Studies of $W$ boson plus jets production in p $\bar{p}$ collisions at $\operatorname{sqrt}(s)=1.96 \mathrm{TeV},[\operatorname{arXiv}: 1302.6508$ [hep-ex] ]. 